\title{
Umfangreiches Kompaktformat: IMR 2021 - Online
}

Sehr geehrte Damen und Herren,

auch in diesem Jahr laden wir Sie ganz herzlich - pandemiebedingt - zu einer kompakten Online-Ausgabe des Intensivkurses Muskuloskelettale Radiologie (IMR) ein.
Save the Date: Der IMR 2021 findet am Samstag, den 11. September 2021 von 08:00-16:15 Uhr statt.

In 5 Highlight-Sessions besprechen wir mit Ihnen die Schwerpunktbereiche „Sprung- gelenk und Fuß“ sowie „Muskel“. Dafür geben renommierte Experten aus Deutschland, Österreich und der Schweiz ihr langjähriges Wissen aus Diagnostik und Therapie zu diesen Themen weiter. \section{SAVE THE DATE: 11. SEPTEMBER}

\section{Wissenschaftliche Leitung: Prof. Dr. Marc Regier}


Bonus: Als Mitglied der Deutschen Röntgengesellschaft erhalten Sie außerdem einen zeitlich begrenzten Zugang zu den Aufzeichnungen auf „conrad“, der digitalen Lernplattform der DRG.

Ich freue mich, an den Erfolg vom letzten Jahr anknüpfen zu können und Sie beim „IMR 2021“ zu einem spannenden und an- regenden Gedankenaustausch im digitalen Format willkommen zu heißen. Sie können sich ab sofort für den „IMR 2021“ online anmelden unter www.msk.drg.de.

Es ist toll und vor allem wichtig, dass die radiologische Fortbildung auch im OnlineGewand gesichert ist - für 2022 wünsche ich mir jedoch, dass wir uns hoffentlich wieder vor Ort austauschen können.

Mit freundlichen Grüßen

Prof. Dr. Marc Regier

Wissenschaftlicher Leiter, Programmgestaltung/Vorsitzender der AG Muskuloskelettale Radiologie 